\title{
微纳米生物活性玻璃诱导牙本质再矿化研究
}

$$
\text { 唐洁吟 }{ }^{1,2} \text {, 王 刚 }{ }^{3,4} \text {, 刘 聪 }{ }^{1,2} \text {, 邹学农 }{ }^{3,4} \text {, 陈晓峰 }{ }^{1,2}
$$

(1. 华南理工大学 材料科学与工程学院, 生物医学工程系, 广州 $510641 ; 2$. 国家人体组织功能重建工程技术研究中 心, 广州 510006); 3. 中山大学附属第一医院 脊柱外科, 广州 510080; 4. 广东省骨科学重点实验室, 广州 510080)

摘 要: 封闭牙本质小管能有效减轻牙齿过敏症。本研究以不同粒径的微纳米生物活性玻璃球(MNBGs)为分散质、 海藻酸钠-磷酸盐缓冲溶液为分散液, 制备了用于牙本质脱敏治疗的 MNBGs 糊剂(MNBGP)。在牙本质切片表面进 行体外矿化并系统评价了糊剂与牙本质的结合性能, 以及糊剂体外诱导牙本质再矿化、封闭牙本质小管的能力。研 究结果表明, 不同粒径 MNBGs 制备的糊剂均能与牙本质界面紧密结合, 粒径较小的 MNBGs 在脱矿牙本质切片表 面分布更加均匀。MNBGP 在人工唾液(AS)中能较好地诱导牙本质再矿化形成磷灰石(HA)以堵塞封闭牙本质小管, 脱矿牙本质切片表面形成的 HA 层随矿化时间延长而增厚, 矿化 $28 \mathrm{~d}$ HA 层的厚度可达到 $5 \sim 10 \mu \mathrm{m}$ 。MNBGs 的尺 寸影响其诱导牙本质再矿化的效果, 当颗粒大小与牙本质小管直径匹配时, MNBGs 可以更好地封闭牙本质小管。 因此, MNBGP 具有良好的治疗牙本质过敏的应用前景。

关 键 词: 牙本质过敏; 微纳米生物活性玻璃; 磷灰石; 再矿化

中图分类号: TQ174 文献标志码: A

\section{Dentin Remineralization Induced by Micro-nano Bioactive Glass Spheres}

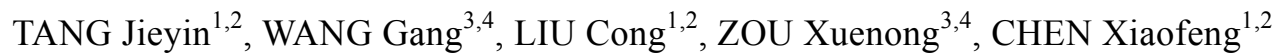

(1. Department of Biomedical Engineering, School of Materials Science and Engineering, South China University of Technology, Guangzhou 510641, China; 2. National Engineering Research Center for Tissue Restoration and Reconstruction, Guangzhou 510006, China); 3. Department of Spine Surgery, The First Affiliated Hospital of Sun Yat-sen University, Guangzhou 510080, China; 4. Guangdong Provincial Key Laboratory of Orthopaedics and Traumatology, Guangzhou 510080, China)

Abstract: Occlusion of dentine tubules may reduce or eliminate dentin hypersensitivity. Here, micro-nano bioactive glass spheres (MNBGs) with various sizes and sodium alginate-phosphate buffer solution were used to prepare bioactive glass pastes (MNBGP) for dentin desensitization. Through mineralization on the surface of dentin samples in vitro, the bonding ability of different pastes with dentin, as well as the ability to induce dentin remineralization and occlude dentin tubules in vitro, was investigated. The results indicated that the MNBGP could be tightly combined with the dentin interface, and the MNBGs with smaller particle sizes were more uniformly distributed on the surface of the demineralized dentin slices. MNBGP could well mineralize in artificial saliva (AS) to form apatite (HA), induce dentin remineralization, and occlude dentin tubules, which resulted in thickening HA layer, formed on the surface of demineralized dentin slices, increased with the prolonged mineralization time, up to $5-10 \mu \mathrm{m}$ after $28 \mathrm{~d}$ dentin

收稿日期：2021-03-12；收到修改稿日期：2021-04-08; 网络出版日期：2021-04-30

基金项目：国家自然科学基金（52003302, 51402108, 32071341）

National Natural Science Foundation of China (52003302, 51402108, 32071341)

作者简介：唐洁吟(1989-), 女, 硕士. E-mail: 1720175343@qq.com

TANG Jieyin (1989-), female, Master. E-mail: 1720175343@qq.com

通信作者：陈晓峰，教授.E-mail: chenxf@scut.edu.cn

CHEN Xiaofeng, professor. E-mail: chenxf@scut.edu.cn 
remineralization. The size of bioactive glass microspheres had a certain impact on its ability to induce remineralization.

When the particle size was equal to the diameter of the exposed dentin tubules, it could better occlude the dentin tubules. All data indicate that MNBGP has potential application for dentin hypersensitivity treatment.

Key words: dentine hypersensitivity; micro-nano bioactive glass spheres; apatite; remineralization

牙本质构成了牙齿的主体硬组织部分，对其功 能的正常行使发挥着极为重要的作用。日常由于龃、 磨耗等均会使牙本质直接暴露在口腔环境中，造成 牙本质过敏, 当受到外界刺激(冷热、酸甜等)时会引 起疼痛，对患者生活产生极大困扰 ${ }^{[1]}$ 。治疗牙本质过 敏可通过封闭牙本质小管降低牙本质的渗透性，或 降低牙髓神经的兴奋性 ${ }^{[2-3]}$ 。目前临床上，使用较多 的脱敏材料包括含氟化物产品、含钾产品、锶盐、 树脂类粘结剂等 ${ }^{[4]}$, 但这类脱敏剂主要与牙本质发 生机械结合而不是化学结合。当暴露于酸性条件或 刷牙时，脱敏剂容易从牙本质表面被去除，疗效并不 持续。

生物活性玻璃因其特有的无机非晶态结构、促 进骨细胞活性、提高生物矿化速度等优点, 能与骨 组织形成化学键合, 同时其降解释放的离子可促进 多种基因表达, 已经成为骨修复领域的重要热点之 - ${ }^{[5-6]}$ 。随着生物活性玻璃机理和应用研究的深入, 由于牙体牙髓组织与骨组织在组成结构上的相似性, 生物活性玻璃在齿科修复领域的应用研究也逐渐增 多 ${ }^{[7]}$ 。2004 年, 研究者首次将生物玻璃 45S5(NovaMin) 添加到牙膏中, 用于治疗牙本质过敏。用含有 NovaMin 的牙亳刷牙后，牙膏中的生物活性玻璃能够附着在 牙本质表面形成羟基磷灰石层 ${ }^{[8-9]}$ 。NovaMin 还替代 传统牙膏中的氧化铝作为摩擦剂, 可降低牙龈出血 的发生率, 抑制牙面菌斑生长 ${ }^{[10]}$ 。除了在牙膏中的应 用, NovaMin 还可以作为抛光膏用于临床牙本质过 敏的治疗和牙齿漂白治疗后脱矿牙釉质的再矿化 ${ }^{[11]}$ 。 Wang 等 ${ }^{[12]}$ 发现用含有 NovaMin 牙亳处理后的牙 本质具有更低的牙本质渗透性, 表面封闭效果也更 好。Vollenweider 等 ${ }^{[13]}$ 比较了使用火焰喷雾合成的 纳米级 45S5 与传统的微米级 $45 \mathrm{~S} 5$, 结合拉曼及 EDX 等分析发现, 45S5 能较好地诱导牙本质再矿化, 且纳米级 $45 \mathrm{~S} 5$ 能更快释放 $\mathrm{Ca}$ 和 $\mathrm{Si}$ 离子, 其再矿化 效果也更显著。Curtis 等 ${ }^{[14]}$ 进一步证明, 相对于熔 融生物活性玻璃在牙本质表面形成磷灰石层, 溶 胶一凝胶结合微波烧结技术制备的纳米生物活性玻 璃能深入牙本质小管形成紧密连接的棒状磷灰石, 更好地封闭牙本质小管。但是上述溶胶一凝胶法制备 的生物活性玻璃颗粒尺寸多在几十微米尺度，且粒 径分布范围较宽，难以进入牙本质小管(管径分布
在 1 3 $\mu \mathrm{m})$; 同时其玻璃实际化学组成与传统 45S5 有较大差异, 无法完全排除其组分对矿化效果的影 响 ${ }^{[15]}$ 。此外, 相关牙本质再矿化研究中, 将生物活 性玻璃粉体沉积在牙本质切片样品正面, 其作为脱 敏材料的操作性及与牙本质初期界面结合效果有待 进一步验证。

近年来，通过溶胶一凝胶法与有机模板法结合 制备得到的微纳米生物活性玻璃( $\mathrm{MNBG}$ ) 以其小尺 寸、单分散及微结构特性, 逐渐受到研究者关注, 与 传统的生物活性玻璃相比, MNBG 形貌及结构尺寸 等可控，具有更高的比表面积和生物活性，能更快 地释放 $\mathrm{Ca}$ 离子及更快的矿化速度 ${ }^{[16-17]}$, 这些特性 对于封闭牙本质小管，缓解牙本质过敏症具有显著 促进作用。本研究以不同尺寸的微纳米生物活性玻 璃球形粉体为原料, 制备出便于操作的生物活性玻 璃糊剂作为牙本质过敏症脱敏材料(MNBGP), 进一 步研究了 MNBGP 与脱矿牙本质切片结合效果、诱 导脱矿牙本质切片体外再矿化及堵塞封闭牙本质小 管的能力。

\section{1 实验方法}

\section{1 微纳米生物活性玻璃的制备}

通过溶胶一凝胶结合模板法制备 MNBGs (摩 尔分数 $80 \% \mathrm{SiO}_{2} 、 16 \% \mathrm{CaO}$ 和 $\left.4 \% \mathrm{P}_{2} \mathrm{O}_{5}\right)^{[18]}$ 。在 $40{ }^{\circ} \mathrm{C}$ 、 磁力搅拌条件下, 首先将模板剂十二胺(DDA)溶 解于一定量去离子水和无水乙醇中; 然后将正硅 酸四乙酯(TEOS)、磷酸三乙酯(TEP)和四水硝酸钙 $(\mathrm{CN})$ 溶液间隔半小时依次滴加到上述溶液中。摚 拌 $3 \mathrm{~h}$ 后, 将所得溶液过夜陈化、洗涤、冷冻干燥, 最后在 $650{ }^{\circ} \mathrm{C}$ 烧结 $3 \mathrm{~h}$ 得到 $\mathrm{MNBG}$ 颗粒。为了制 备不同粒径的 MNBGs, 实验中改变模板剂 DDA、前驱体 TOES 的用量(表 1)制备出不同粒径 的 MNBGs，分别简称为 MNBGs-1、MNBGs-2 和 MNBGs-3。

\section{2 牙本质切片样品的制备}

在广州医科大学附属口腔医院领面外科收集了 健康人体第三磨牙 80 颗, 牙齿相关实验均通过该院 的伦理审查。将收集到的牙齿及时处理清洗干净, 
表 1 不同粒径 MNBGs 的理论、实际化学组分和试剂用量

Table 1 Theoretical and measured chemical composition, reagent dosage of MNBGs with different particle sizes

\begin{tabular}{ccccc}
\hline \multirow{2}{*}{ Sample } & \multicolumn{2}{c}{$\mathrm{SiO}_{2}: \mathrm{CaO}: \mathrm{P}_{2} \mathrm{O}_{5}$ (molar ratio) } & DDA/g & $\mathrm{TEOS} / \mathrm{mL}$ \\
\cline { 2 - 5 } & Theoretical & Measured & 3 & 8 \\
MNBGs-1 & \multirow{2}{*}{$80: 16: 4$} & $87.637: 12.363: 0$ & 6 & 16 \\
MNBGs-2 & & $86.289: 13.696: 0.015$ & 6 & 24 \\
MNBGs-3 & $89.071: 10.875: 0.054$ & & 6 \\
\hline
\end{tabular}

然后用 $0.1 \%(w / v)$ 的鹿香草酚溶液浸泡, $4{ }^{\circ} \mathrm{C}$ 保存备 用。用硬组织切割机沿轴向切割牙齿, 打磨制备成 牙本质切片样品 $(5 \mathrm{~mm} \times 5 \mathrm{~mm} \times 2 \mathrm{~mm})$, 然后超声清 洗置于鹰香草酚溶液待用。考虑到牙本质结构中含 有大量无机组分碳酸差基磷灰石( $\mathrm{HCA})$, 对制备的 牙本质切片样品用乙二胺四乙酸(EDTA)酸蚀去除 其无机矿物, 可以更真实地反映材料体外诱导牙本 质矿化的能力 ${ }^{[19]}$ 。

\section{3 生物活性玻璃矿化材料在脱钙牙本质 切片样品上固定}

将不同粒径的 MNBGs 微球按粉液比为 $200 \mathrm{mg} / \mathrm{mL}$ 的比例分散到海藻酸钠-磷酸盐溶液中, 超声处理使其进一步分散均匀, 最后得到生物活性 玻璃糊剂(MNBGP)。不同粒径 MNBGs 制备的糊剂 分别称为 MNBGP-1、MNBGP-2、MNBGP-3。

对预备好的脱矿牙本质切片样品进行冲洗、灭 菌、干燥, 再将少量 MNBGP 糊剂涂覆于脱矿牙本 质切片样品上, 自然晾干半小时, 再用 $1 \mathrm{~mL}$ 去离子 水冲洗 3 次，晾干。

\section{4 脱矿牙本质切片在人工唾液中再矿化}

用 $\mathrm{GAL}^{[20]}$ 提出的人工唾液 (AS) 配方, 将涂覆 有 MNBGP 的脱矿牙本质切片样品浸泡在 $10 \mathrm{~mL}$ $\mathrm{AS}$ 溶液中, $37{ }^{\circ} \mathrm{C}$ 恒温恒湿养护箱中静置; 并将没 有涂覆糊剂的牙本质切片样品进行类似处理设为对 照组, 每组设 3 平行样。分别矿化 1、7、14、28 d 后 取出样品, 用乙醇、水交替清洗三次, $37{ }^{\circ} \mathrm{C}$ 烘干, 进 一步测试脱矿牙本质切片样品, 评价不同 MNBGP 体外诱导牙本质再矿化形成羟基磷灰石的能力。

\section{5 测试与表征}

采用高分辨场发射扫描电子显微镜(FE-SEM, Merlin, ZEISS)观察样品的表面形貌，同时结合 X 射 线能谱分析(EDS)测定材料的化学组成。采用场发射 透射电子显微镜(TEM, JEM-2100HR, JEOL)观察样 品的微观结构。选取扫描电镜图片中颗粒(至少 100 个), 采用 ImageJ 软件进行测量, 统计其粒径结果。通过 射线衍射分析仪 (XRD, $X^{\prime}$ Pert ${ }^{3}$ Powder $X$, PANalytical)测定样品的物相组成。采用傅里叶变换
红外光谱仪(FT-IR, Vector33, Bruker)测定样品的化学 结构。

\section{2 结果与讨论}

\subsection{MNBG 形貌和结构}

通过改变模板剂 DDA 和前驱体 TEOS 用量, 成功制备出不同粒径的微纳米生物活性玻璃微球 (MNBGs), 图 1 为不同粒径 MNBGs 的 SEM 照片、 TEM 照片和粒径分布图。从 SEM 照片(图 1(a1 c1)) 中可以观察到, 三种不同粒径的 MNBGs 颗粒均呈 规则的球形; 微球表面表现出小颗粒堆积的粗䊁结 构。从 TEM 照片(图 1(a2 c2)) 可以观察到, 不同粒 径的 MNBGs 为实心结构, 且均具有良好的分散性。 从粒径分布图(图 1(a3 c3))可以观察到, 三种 MNBGs 的粒径分布均较窄, 说明三种 MNBGs 尺寸分布较 均匀, 平均粒径分别为 300、700 和 $1100 \mathrm{~nm}$ 。

进一步分析制备的 MNBGs 物相组成和化学结 构, 图 2 为三种 MNBGs 的 XRD、FT-IR 图谱。从 XRD 图谱可以观察到(图 2(a))三种 MNBGs 的峰形 相似, 在 $2 \theta=23^{\circ}$ 附近呈现弥散衍射峰, 这与非晶态 硅酸盐的特征衍射峰相对应 ${ }^{[21]}$, 结果表明改变粒径 对其非晶态结构并无显著影响。从 FT-IR 图谱(图 2(b))可以观察到, 不同粒径的 MNBGs 图谱同样具 有相似性，在 1090、800 和 $475 \mathrm{~cm}^{-1}$ 处的吸收带分 别与 $\mathrm{Si}-\mathrm{O}-\mathrm{Si}$ 的非对称伸缩振动、对称伸缩振动和 对称弯曲振动峰对应 ${ }^{[22]}$, 且三种大小颗粒的峰位均 未发生显著的偏移，表明 MNBGs 的化学结构也不 受颗粒粒径变化的影响。

\subsection{MNBGs 与脱矿牙本质切片样品结合}

牙本质切片酸蚀(EDTA 处理)前后的 SEM 照片 如图 3, 能谱分析结果如表 2。可以观察到(图 3(a)), 酸蚀前牙本质切片样品的表面牙本质小管暴露, 表 面留下少量切割凹痕, 制备的牙本质切片牙本质小 管的内径约 1 2 $\mu \mathrm{m}$, 而自然牙本质-釉质界面处正 常小管直径为 $0.5 \sim 0.9 \mu \mathrm{m}^{[15]}$, 其直径差异是由于切 片样品切割时更靠近牙齿颈部，稍远于牙冠; EDS 能谱分析结果表明酸蚀前牙本质切片表面含有较多 

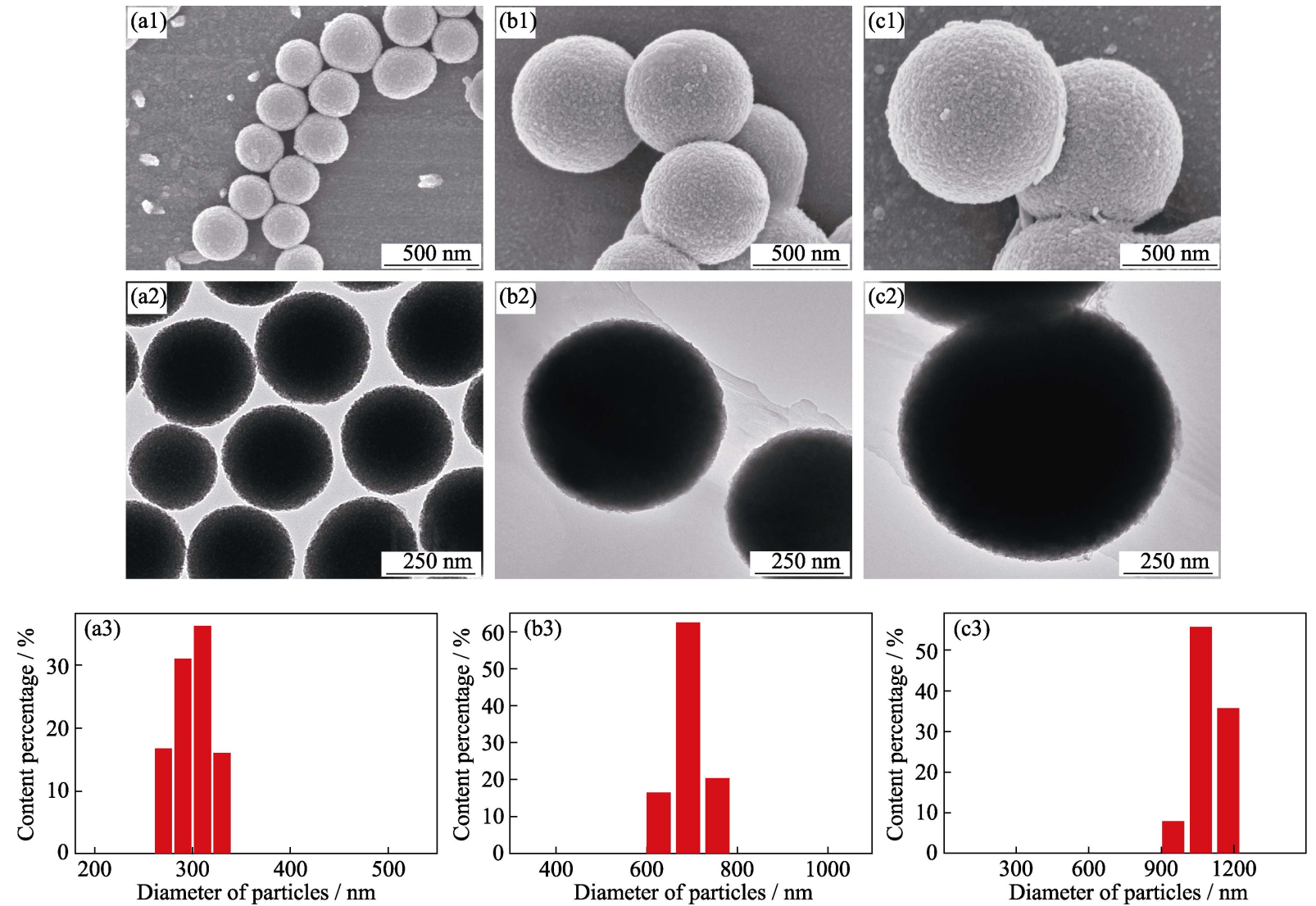

图 1 不同粒径的微纳米生物活性玻璃球的 $\operatorname{SEM}(\mathrm{a} 1 \sim \mathrm{c} 1)$ 、 TEM(a2 c 2$)$ 照片和粒径分布图 $(\mathrm{a} 3 \sim \mathrm{c} 3)$

Fig. 1 SEM (a1-c1), TEM (a2-c2) images and particle size distributions (a3-c3) of MNBGs with different particle sizes
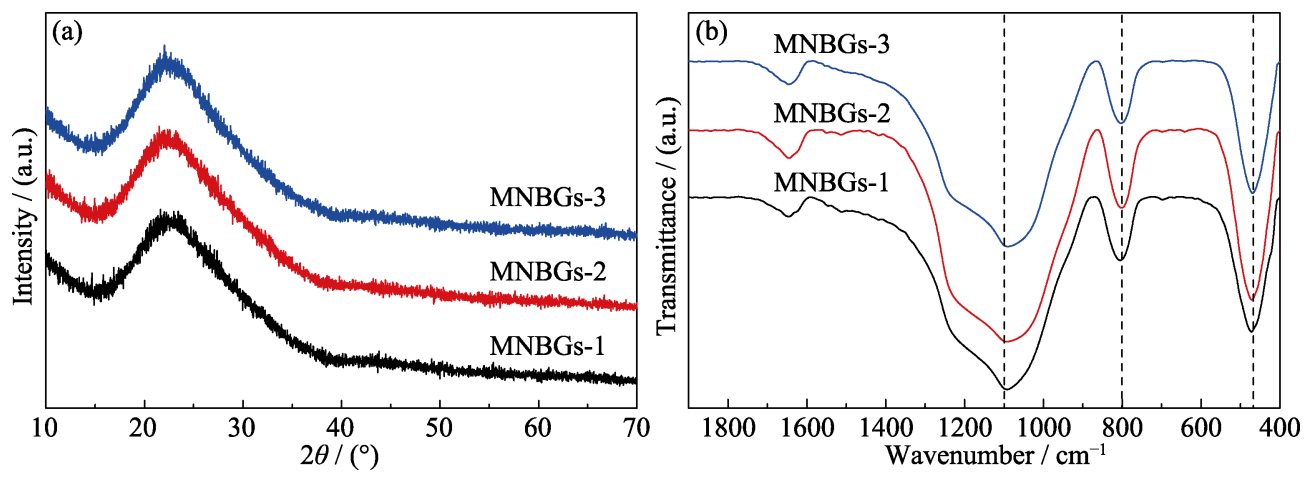

图 2 三种微纳米生物活性玻璃球的 XRD 图谱(a)和 FT-IR(b)图谱

Fig. 2 XRD patterns (a) and FT-IR spectra (b) of MNBGs
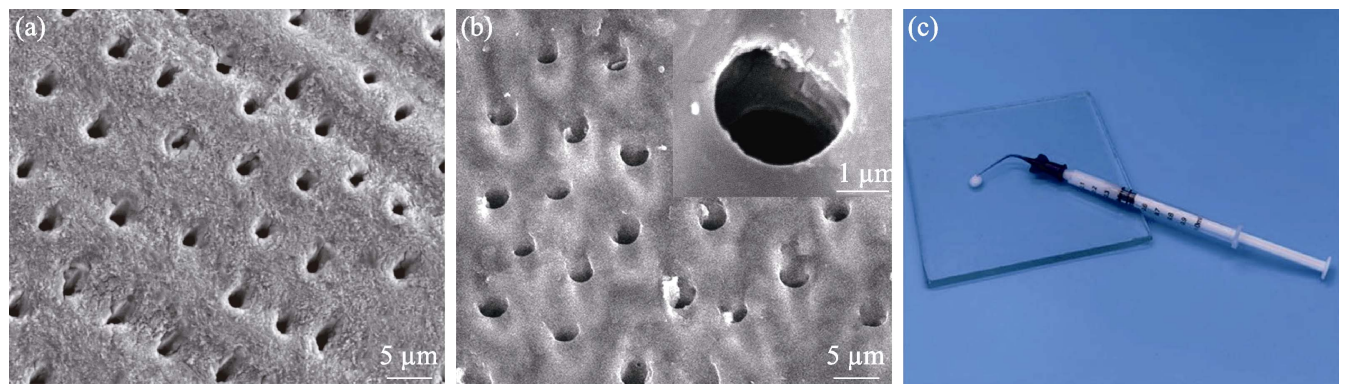

图 3 牙本质切片样品酸蚀前(a)后(b)的 SEM 照片(插图为牙本质小管放大照片)及生物活性玻璃糊剂实物照片(c)

Fig. 3 SEM images of the dentin surface without (a) and with (b) EDTA-etching, magnified photo of dentin tubules (insert in (b)), and photo of bioactive glass paste (c) 
表 2 牙本质切片表面 EDTA 酸蚀前后各元素 含量(摩尔分数)及钙磷比

Table 2 Chemical components (molar percent) and $\mathrm{Ca} / \mathrm{P}$ ratio on the surface of dentin before and after EDTA etching

\begin{tabular}{lccrrc}
\hline \multicolumn{1}{c}{ Sample } & $\mathrm{C} / \%$ & $\mathrm{O} / \%$ & $\mathrm{Ca} / \%$ & $\mathrm{P} / \%$ & $\mathrm{Ca} / \mathrm{P}$ \\
\hline Without etching & 0 & 65.77 & 20.26 & 13.97 & 1.45 \\
With etching & 66.82 & 32.99 & 0.05 & 0.14 & 0.35 \\
\hline
\end{tabular}

钲、磷元素, $\mathrm{Ca} / \mathrm{P}$ 比值为 1.45 (如表 2), 其比值与羟 基磷灰石的 $\mathrm{Ca} / \mathrm{P}$ 理论值 (1.67)接近 ${ }^{[19,23]}$ 。经 EDTA 酸蚀处理后(图 3(b)), 切片表面变得更加光滑, 小管 直径略微增加, 这是由于 EDTA 酸蚀脱去牙本质小 管壁的无机矿物, 仅剩少量胶原纤维等有机物(如 图 3(b)插图), 牙本质小管壁变薄, 进而增大牙本质 小管直径。对比酸蚀后牙本质切片 EDS 能谱分析, 发现切片表面钙、磷含量极低, 对应 $\mathrm{Ca} / \mathrm{P}$ 比值仅为 0.35 , 远小于 1.67 , 说明 EDTA 酸蚀处理对牙本质切 片表面的无机矿物成功完成了脱矿。

制备的生物活性玻璃糊剂(MNBGP) (图 3(c)) 具 有良好的操作性。用 MNBGP 涂覆脱矿牙本质切片 样品表面的 SEM 照片如图 4 所示。从图中可以观察 到, 由于 MNBGP 液相含有一定量海藻酸钠, 使得 糊剂具有黏性, 促进 MNBGs 更好地粘附在脱矿牙 本质切片表面。图 4 显示, 粒径较小的 MNBGs 能
更均匀分布在脱矿牙本质切片表面(图 4(a)), 随着 MNBGs 粒径增加, 逐渐形成团聚体, 产生的空隙也 相应增大, MNBGs 在脱矿牙本质切片表面的分布也 更不均匀(图 4(c))。此外, 海藻酸钠富含的羧基能够 与 $\mathrm{Ca}^{2+}$ 发生离子交联, 形成网络结构 ${ }^{[24-25]}$, 因此, 海藻酸钠能够与生物活性玻璃等相互作用, 同时促 进矿化的磷灰石层与牙本质切片结合。

进一步对 MNBGP 涂覆前后以及用去离子水冲 洗后的脱矿牙本质切片样品进行全反射-红外光谱 (ATR-FTIR)分析(图 5), 由图可知, 未涂覆 MNBGP 的脱矿牙本质切片样品(对照组)的 ATR-FTIR 光谱 无明显的 P-O 吸收峰, 表明酸蚀处理后, 切片表面 无机组分基本除去, 结果也与 EDS 能谱分析对应; 与对照组(涂覆 MNBGP 处理)相比, 涂覆后脱矿牙 本质切片表面的 ATR-FTIR 光谱在 $800 、 1090 \mathrm{~cm}^{-1}$ 处均出现了明显的 $\mathrm{Si}-\mathrm{O}-\mathrm{Si}$ 特征吸收峰 ${ }^{[26]}$; 进一步 用离子水冲洗处理, 脱矿牙本质切片表面仍然保留 $\mathrm{Si}-\mathrm{O}-\mathrm{Si}$ 特征对应吸收峰, 且峰位无明显偏移, 表明 MNBGP 与脱矿牙本质切片仍结合紧密, MNBGs 能 较好地黏附。进一步对图 5(b, c) 分析, 脱矿牙本质 切片表面的特征峰强度 $\left(800 、 1090 \mathrm{~cm}^{-1}\right)$ 经去离子水 冲洗后较冲洗前强度降低, 可能是由于部分 MNBGs 被冲掉从而减弱了特征峰强度。
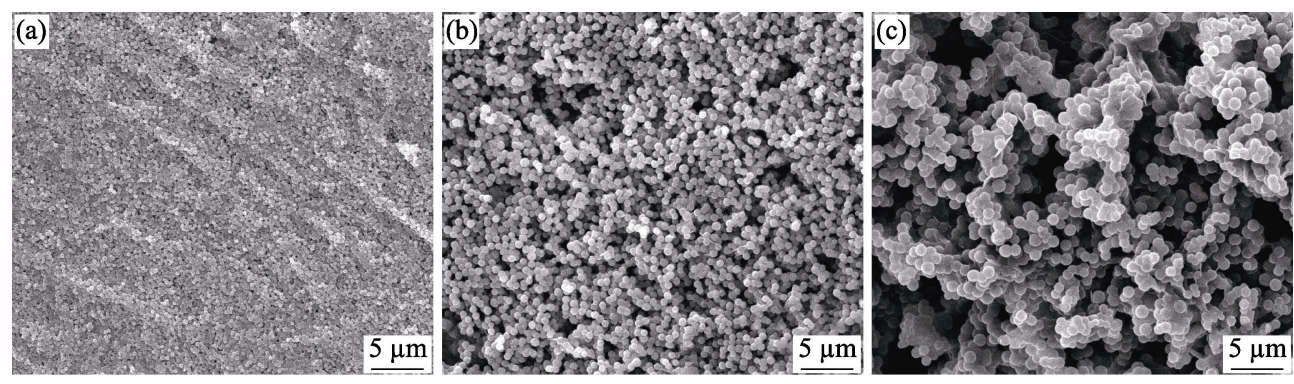

图 4 不同 MNBGP 涂覆的脱矿牙本质切片表面 SEM 照片

Fig. 4 SEM images of demineralized dentin slices coating with different MNBGP (a) MNBGP-1; (b) MNBGP-2; (c) MNBGP-3
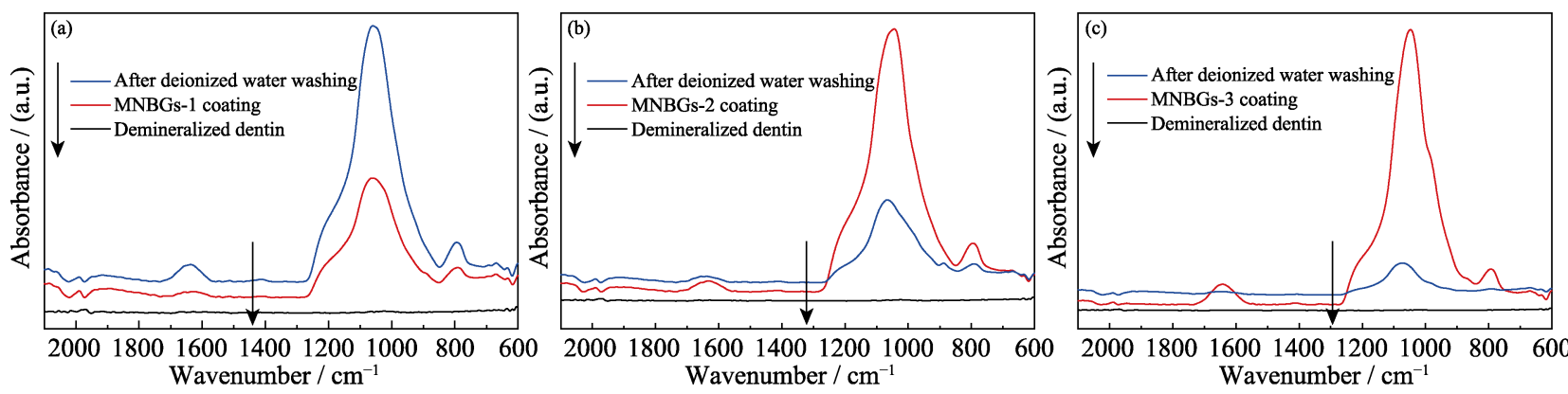

图 5 脱矿牙本质切片表面涂覆不同 MNBGP 前后, 以及去离子水冲洗后的全反射一红外光谱

Fig. 5 ATR-FTIR spectra of demineralized dentin surface before and after coating with MNBGP, and after rinsing with water

(a) MNBGP-1; (b) MNBGP-2; (c) MNBGP-3 


\subsection{MNBGs 体外诱导牙本质再矿化}

在人工唾液中对未涂覆糊剂(对照组)和涂覆不 同 MNBGP 糊剂(实验组)的脱矿牙本质切片进行体 外磷灰石形成能力评价, 脱矿牙本质切片表面矿化 不同时间的 SEM 照片如图 6 所示。从图 6(a1 a4) 可以观察到，对照组样品矿化后，由于表面的无机 矿物晶体已经酸蚀脱去, 无法吸附 $\mathrm{Ca}^{2+}$ 和 $\mathrm{PO}_{4}{ }^{3-}$, 进 而阻碍矿化结晶形成类羟基磷灰石 ${ }^{[27]}$, 矿化前后牙 本质小管仍呈开放状态，表明脱矿后牙本质切片无 法自行矿化形成足够的羟基磷灰石以封闭牙本质小 管，这也与天然过敏牙本质较难自矿化缓解牙本质 症状相一致。从图 6(b1 d1) 可以观察到, 脱矿牙本 质切片表面涂覆 MNBGP 后, 部分 MNBGs 颗粒可 以直接填充并堵塞开放的牙本质小管，隔绝外界刺 激; 随着脱矿牙本质切片在人工唾液中矿化时间延 长, $\mathrm{MNBGs}$ 在人工唾液进行离子交换, $\mathrm{Ca}^{2+} 、 \mathrm{PO}_{4}{ }^{3-}$
局部达到饱和, 促进 HA 在 MNBGs 和脱矿牙本质 切片表面沉积(图 6(b2 d2)和图 6(b3 d 3 )), 矿化到 第 $28 \mathrm{~d}$ 时, 糊剂涂覆的脱矿牙本质切片表面基本被 HA 完全覆盖(图 6(b4 d4))。矿化前期, MNBGs 颗粒 物理充填开放的牙本质小管, 阻隔牙本质小管与外 界环境，从而保护牙髓组织及牙髓神经; 在人工唾 液中矿化一段时间后, MNBGs 通过化学沉积 HA 层 彻底隔绝牙本质与人工唾液。比较不同 MNBGP 糊 剂处理组(图 6(b d))照片, 可以观察到不同 MNBGP 对牙本质小管封闭及诱导牙本质再矿化的能力有差 异，其顺序对应: MNBGP-2>MNBGP-1>MNBGP-3。 其顺序不完全与 MNBGs 粒径大小对应，推测 MNBGP 诱导牙本质再矿化的能力受玻璃颗粒直径 和玻璃颗粒自身矿化活性的综合作用，一方面只有 颗粒大小与暴露的牙本质小管口径相匹配(颗粒大小 与牙本质小管直径相近或略小于小管直径)才能更好
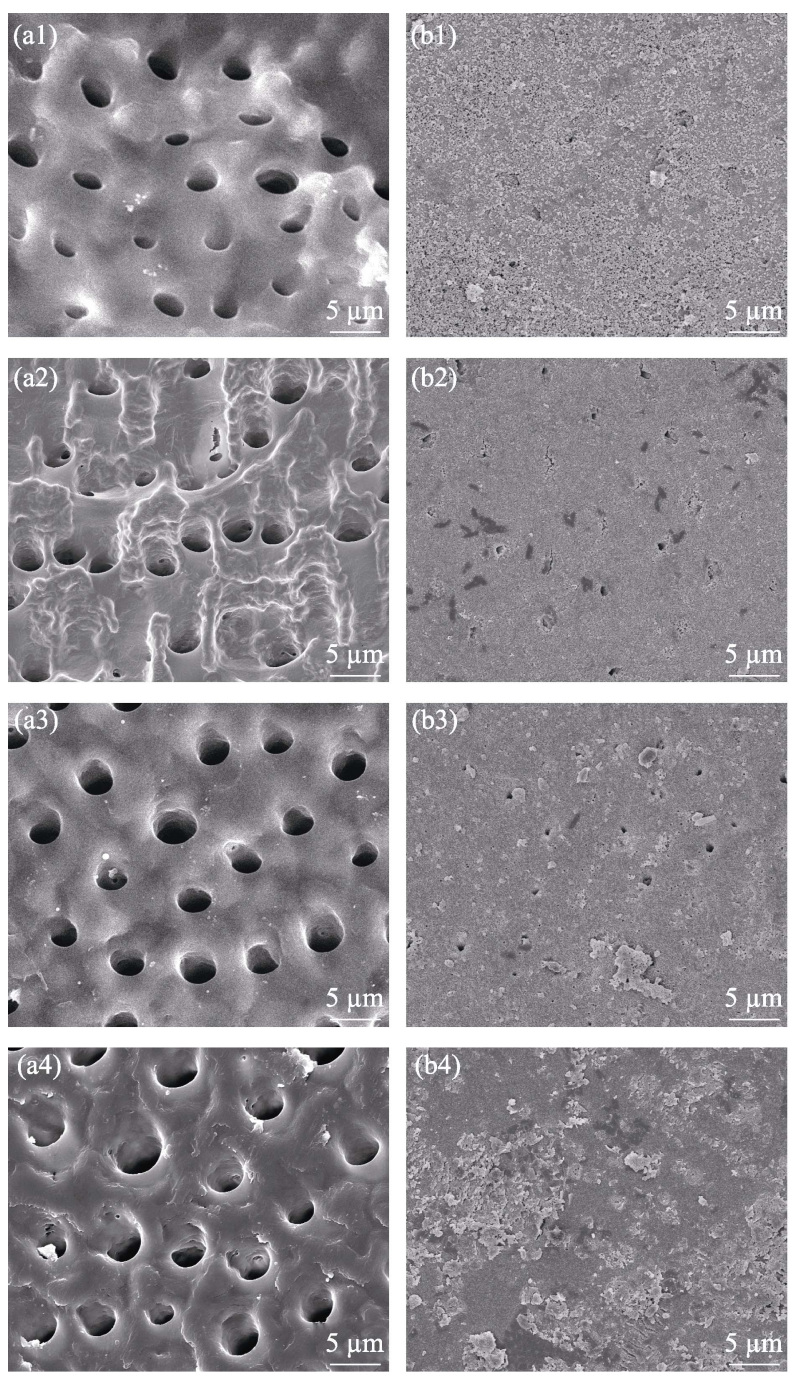
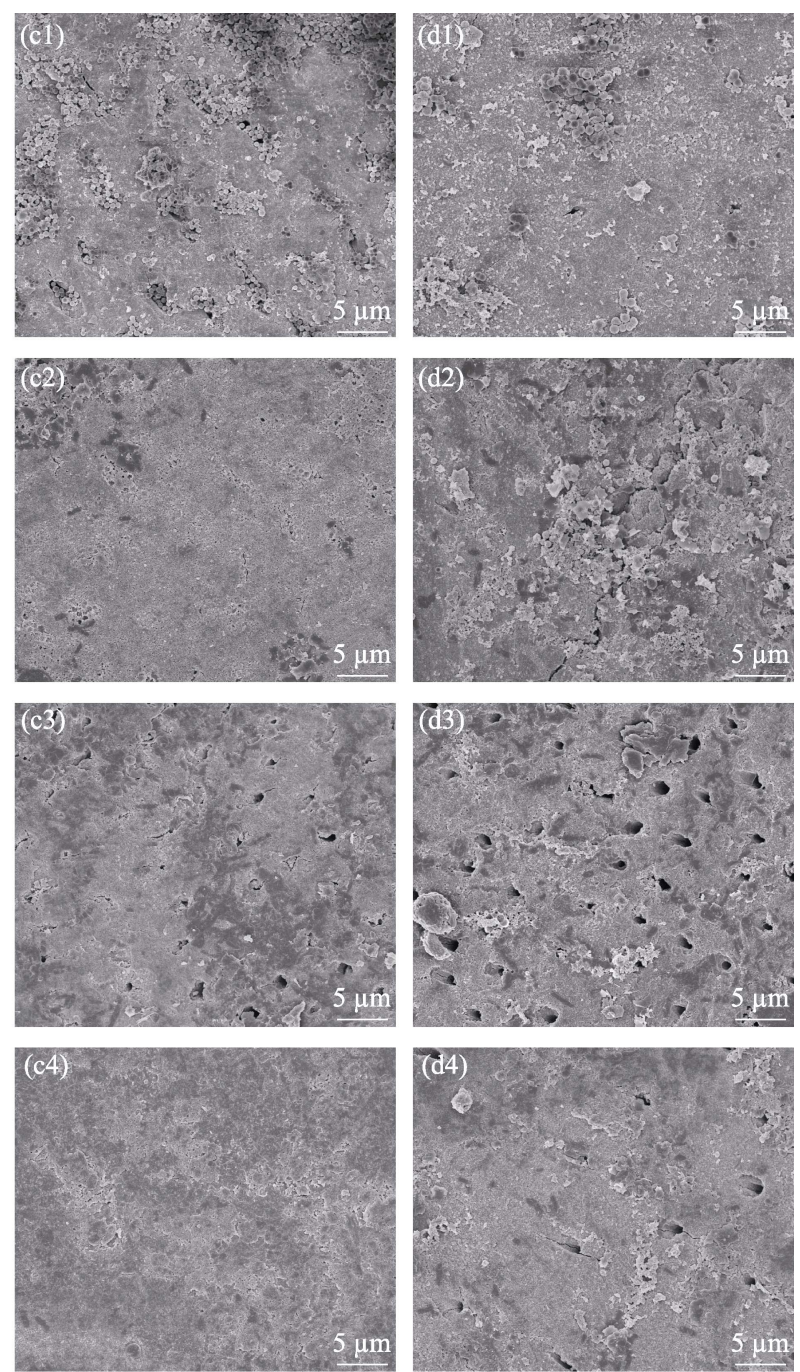

图 6 未经材料处理(对照组)(a)和涂覆 MNBGP-1(b)、MNBGP-2(c)、MNBGP-3(d)的脱矿牙本质切片 在人工唾液中浸泡 $1 \mathrm{~d}(\mathrm{a} 1 \sim \mathrm{d} 1) 、 7 \mathrm{~d}(\mathrm{a} 2 \sim \mathrm{d} 2) 、 14 \mathrm{~d}(\mathrm{a} 3 \sim \mathrm{d} 3)$ 和 $28 \mathrm{~d}(\mathrm{a} 4 \sim \mathrm{d} 4)$ 后的表面 SEM 照片

Fig. 6 SEM images of the surfaces of demineralized dentin slices without (a) (control) and with treatment by MNBGP-1 (b), MNBGP-2 (c), and MNBGP-3 (d) after soaking in AS for $1 \mathrm{~d}(\mathrm{a} 1-\mathrm{d} 1), 7 \mathrm{~d}(\mathrm{a} 2-\mathrm{d} 2), 14 \mathrm{~d}(\mathrm{a} 3-\mathrm{d} 3)$ and $28 \mathrm{~d}(\mathrm{a} 4-\mathrm{d} 4)$ 
地物理堵塞以封闭牙本质小管; 另一方面生物活性 玻璃自身矿化活性越高, 化学作用沉积的 HA 更多, 其封闭效果也越好。

图 7 为脱矿牙本质切片样品在人工唾液中矿化 不同时间后的纵截面(平行牙本质小管方向)SEM 照片，对照组的脱矿牙本质切片的截面照片显示, 牙本质小管内壁及截面边缘均没有形成明显矿化物, 表明切片脱矿后在人工唾液中无法自发矿化封闭牙 本质小管。与对照组相比, 经 MNBGP 涂覆过的脱 矿牙本质切片截面边缘的牙本质小管内充填有 MNBGs 颗粒，牙本质小管被紧密堵塞，通过小管内 外 MNBGs 进一步的矿化作用, 牙本质小管管口处沉 积一层 HA, 进一步封闭并隔绝外界刺激; 随矿化时 间进一步延长, MNBGP 涂覆过的脱矿牙本质切片
表面沉积更厚的 HA层，矿化 $28 \mathrm{~d}$ (图 7 (a4, b4, c4)), HA 层最厚可达 5 10 $\mu \mathrm{m}^{[28]}$ 。

图 8 为脱矿牙本质切片样品矿化 $28 \mathrm{~d}$ 后的表面 能谱扫描分析。矿化 $28 \mathrm{~d}$ 后, 脱矿牙本质切片样品 表面 Ca、P 元素均有所增加, 且实验组显著高于对 照组, 其中玻璃颗粒尺寸较小的组(MNBGP-1 和 MNBGP-2 处理组)钙、磷元素含量变化更显著, 具 体 Ca、P 参数见表 3 。结果表明 MNBGP-1 和 MNBGP-2 组 $\mathrm{Ca} 、 \mathrm{P}$ 含量明显提高, 其 $\mathrm{Ca} / \mathrm{P}$ 比值也最接近天然 牙本质的 1.67 , 说明涂覆 MNBGP 糊剂能较好地诱 导脱矿牙本质的再矿化, 粒径较小的 MNBGs 组糊 剂涂覆脱矿牙本质切片后, 其矿化形成的 HA 晶体 结构更接近天然牙本质。值得注意的是, 矿化后对 照组的 $\mathrm{Ca} 、 \mathrm{P}$ 含量仍较少, 但是其 $\mathrm{Ca} / \mathrm{P}$ 比值同样
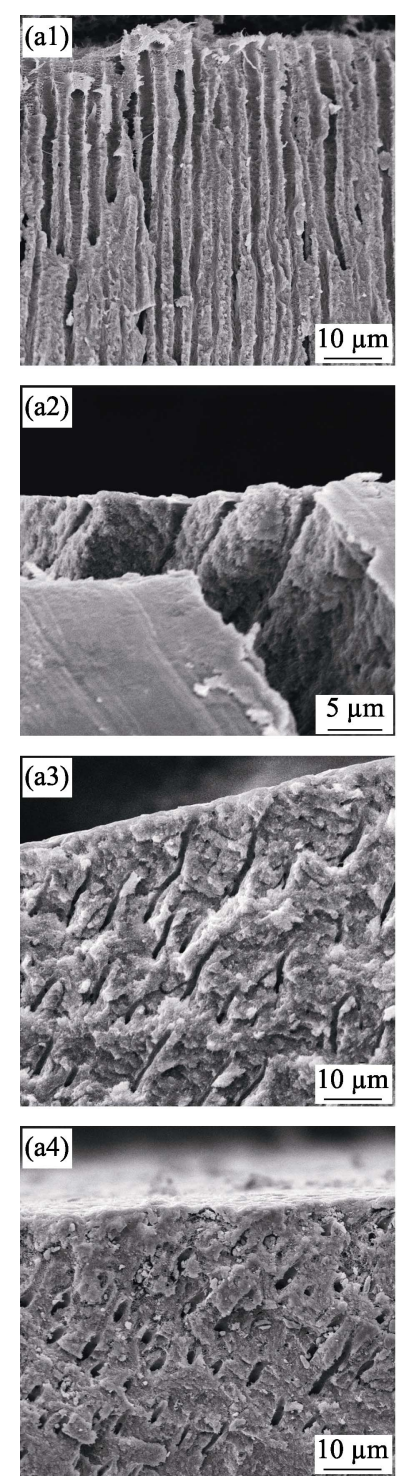
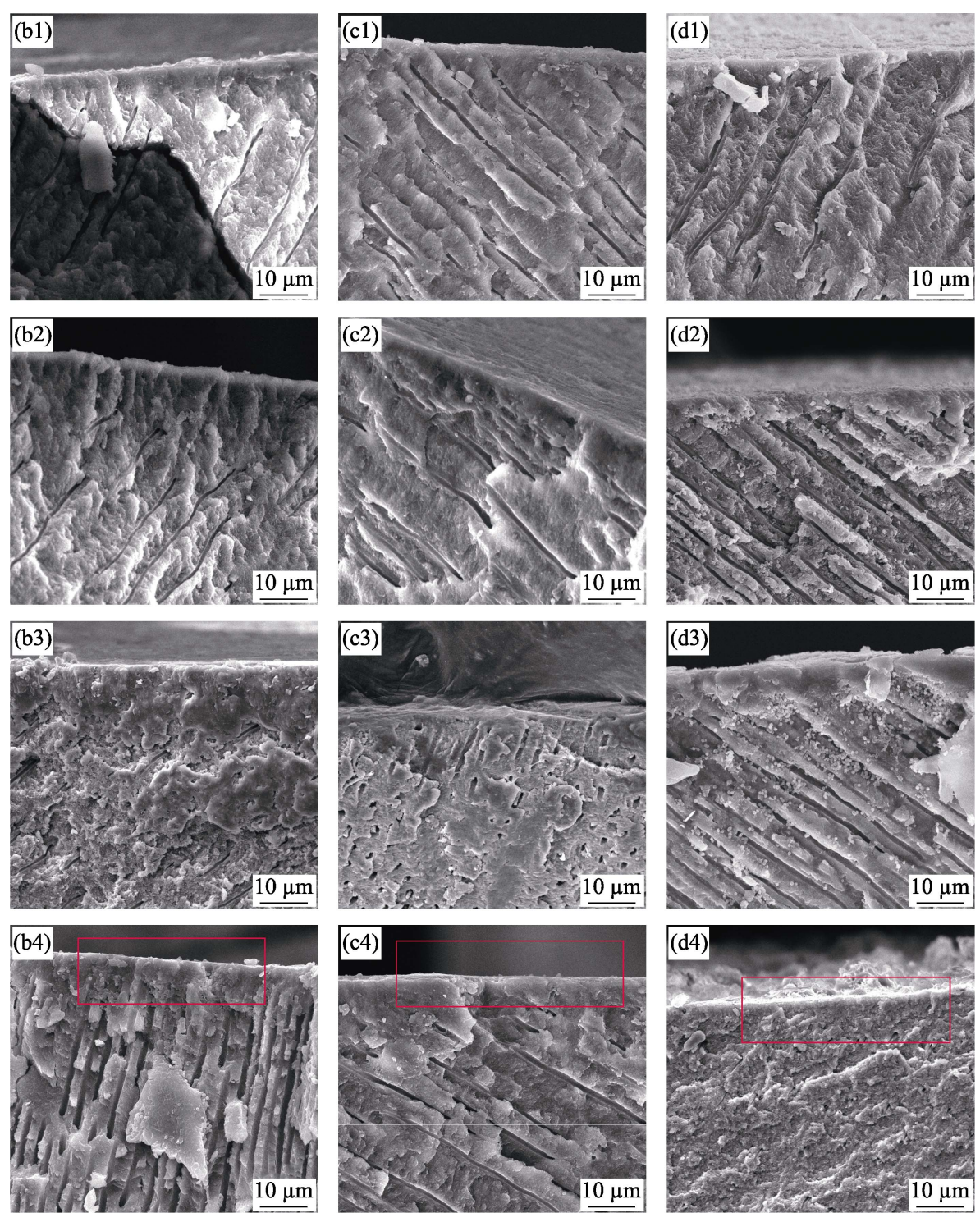
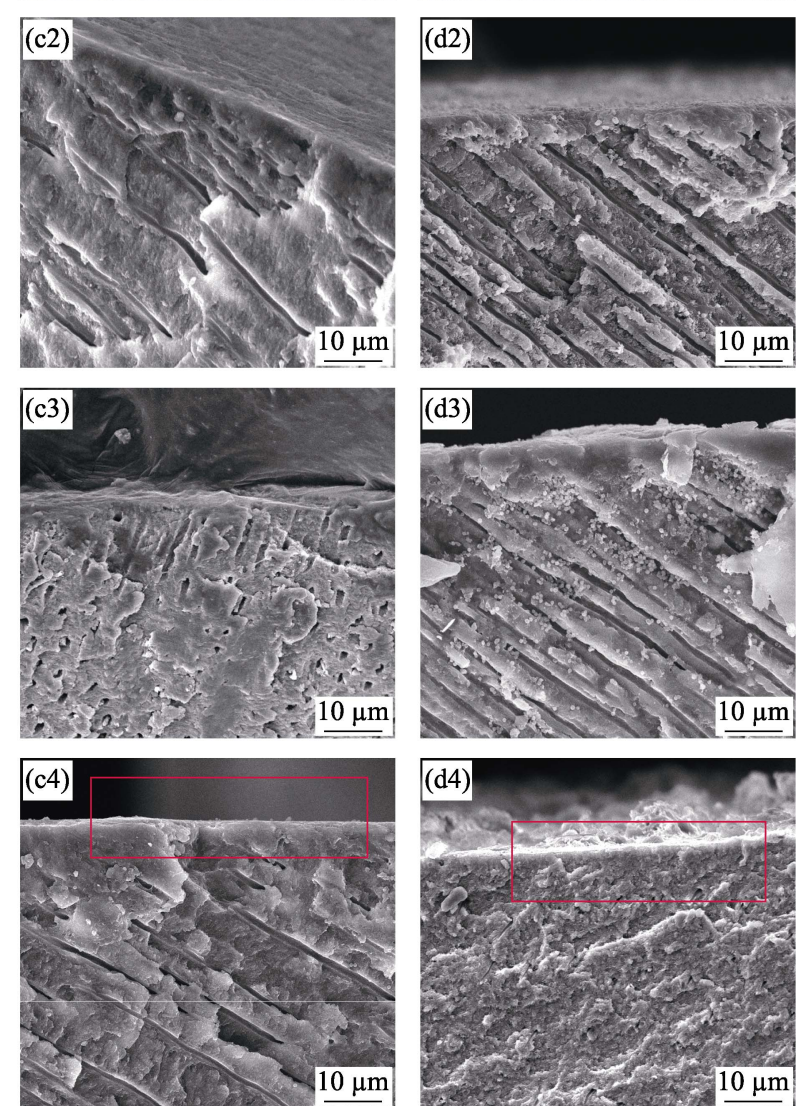

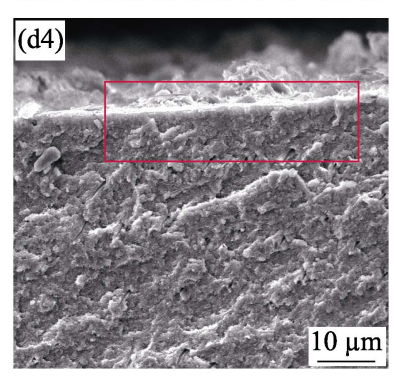

图 7 未经材料处理(a)和涂覆 MNBGP-1(b)、MNBGP-2(c)、MNBGP-3(d)的脱矿牙本质切片在人工唾液中浸泡 $1 \mathrm{~d}(\mathrm{a} 1 \sim \mathrm{d} 1) 、 7 \mathrm{~d}(\mathrm{a} 2 \sim \mathrm{d} 2) 、 14 \mathrm{~d}(\mathrm{a} 3 \sim \mathrm{d} 3)$ 和 $28 \mathrm{~d}(\mathrm{a} 4 \sim \mathrm{d} 4)$ 后的纵截面的 SEM 照片

Fig. 7 SEM images of the longitudinal section of demineralized dentin samples without (a) (control) and with treatment by MNBGP-1 (b), MNBGP-2 (c), and MNBGP-3 (d) after soaking in AS for $1 \mathrm{~d}(\mathrm{a} 1-\mathrm{d} 1), 7 \mathrm{~d}(\mathrm{a} 2-\mathrm{d} 2), 14 \mathrm{~d}(\mathrm{a} 3-\mathrm{d} 3)$ and 28 d (a4-d4) 

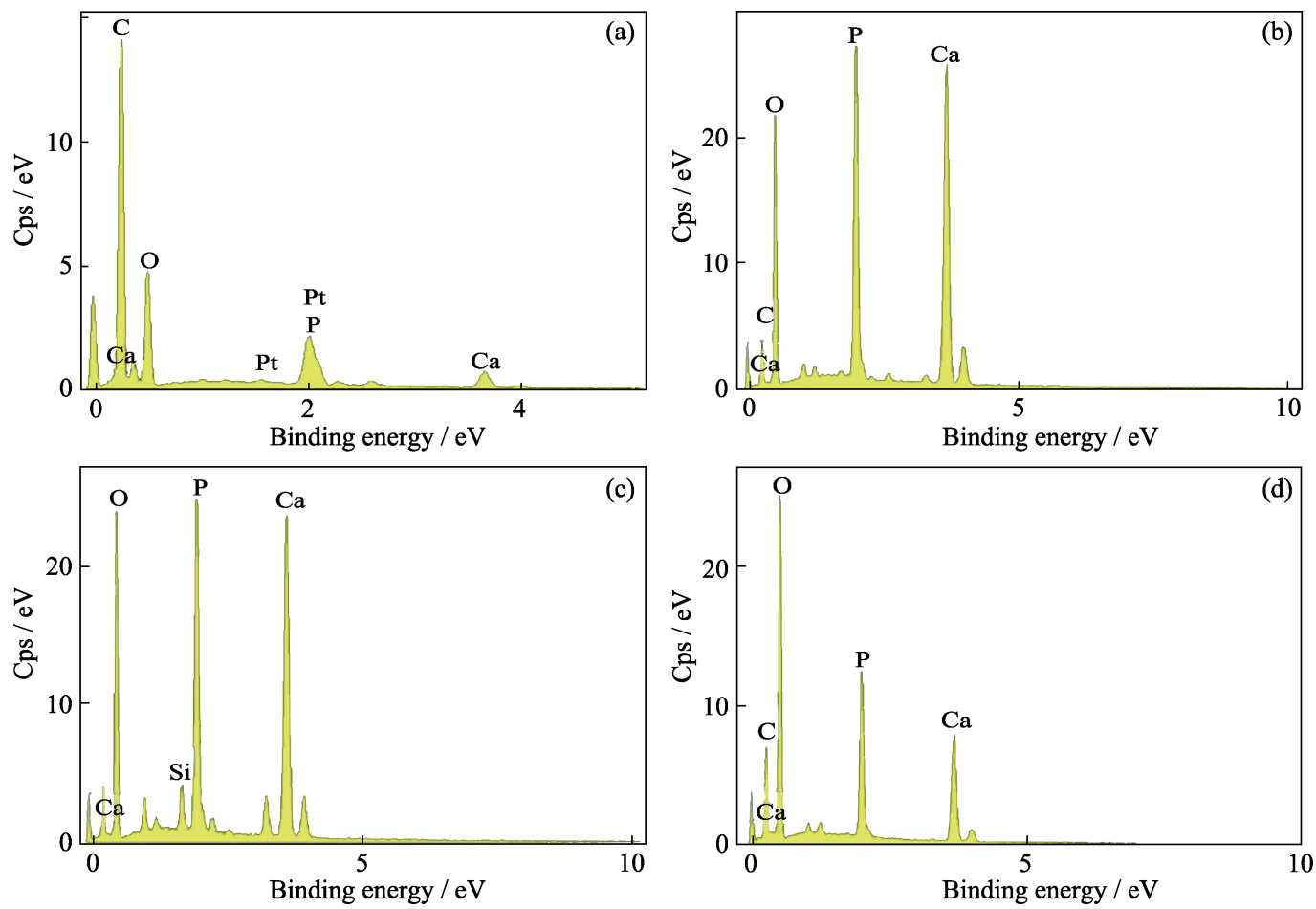

图 8 对照组(a)和 MNBGP-1(b)、MNBGP-2(c)、MNBGP-3(d)涂覆的脱矿牙本质切片在 人工唾液中矿化 $28 \mathrm{~d}$ 表面的 $\operatorname{EDS}$ 能谱分析

Fig. 8 EDS analyses of the surface of demineralized dentin slices without (a) (control) and with treatment by MNBGP-1 (b), MNBGP-2 (c), and MNBGP-3 (d) after soaking in AS for $28 \mathrm{~d}$

表 3 脱矿牙本质切片表面钻、磷元素含量 (摩尔分数)及钻磷比

Table 3 Chemical components ( molar percent) and $\mathrm{Ca} / \mathrm{P}$ ratio in molar on the surface of remineralized dentin

\begin{tabular}{lrrr}
\hline \multicolumn{1}{c}{ Sample } & $\mathrm{Ca} / \%$ & $\mathrm{P} / \%$ & $\mathrm{Ca} / \mathrm{P}$ \\
\hline Control & 2.49 & 1.82 & 1.37 \\
MNBGP-1 & 16.64 & 11.69 & 1.42 \\
MNBGP-2 & 18.37 & 12.97 & 1.42 \\
MNBGP-3 & 6.16 & 6.12 & 1.01 \\
\hline
\end{tabular}

与天然牙本质的 $\mathrm{Ca} / \mathrm{P}$ 比近似，表明在人工唾液环境 下，牙本质自身也可能发生少量再矿化，但是由于 磷灰石沉积过少无法通过 SEM 直接观察到, 这也 与前面对照组矿化 $28 \mathrm{~d}$ 的 SEM 照片仍显示大量暴 露牙本质小管对应。

对脱矿牙本质切片矿化 $28 \mathrm{~d}$ 的表面物相结构进 行 XRD 分析(如图 9)。从图谱中可以看出，与天然 牙本质对比, 脱矿牙本质表面的 HA 特征峰强度降 低, 衍射峰数量减少, 特别是(002)、(211)、(112)和 (202)晶面衍射峰。矿化 $28 \mathrm{~d}$ 后, 对照组(没有糊剂 处理)及糊剂处理过的脱矿牙本质切片样品表面均 出现 HA 特征衍射峰: 对照组 HA 特征衍射峰较少, 仅少量对应(002)、(211)和(112) 晶面且峰强较小; 涂 覆不同 MNBGP 糊剂后的脱矿牙本质切片表面则出 现更多 HA 特征衍射峰: 对应(002)、(211)、(112)、(300)、 (202)、(310)、(222)、(213)和(004)晶面 ${ }^{[29], ~}$ 衍射峰

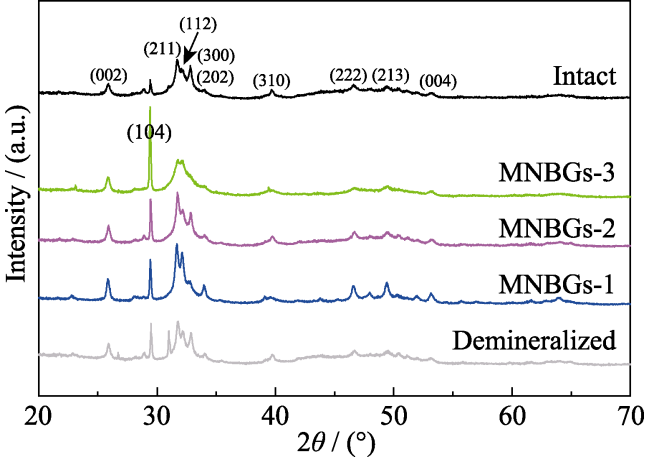

图 9 天然牙本质、脱矿牙本质和在人工唾液中浸泡 $28 \mathrm{~d}$ 的脱矿牙本质切片样品表面的 XRD 图谱

Fig. 9 XRD patterns of the surface of intact dentin, demineralized dentin and slices without treatment (control) and being treated with MNBGP after being soaked in AS for $28 \mathrm{~d}$

显著增强，其峰位与峰强也更接近天然牙本质，表明 脱矿牙本质切片经 MNBGP 处理和一定时间(28 d) 矿 化可形成晶体结构类似天然牙本质的 HA 层。值得 注意的是，生物玻璃粒径较小的 MNBGP-1 和 MNBGP-2 组的衍射峰强度最大, 说明 MNBGP-1 和 MNBGP-2 组处理脱矿牙本质切片诱导形成的 HA 数量更多, 其结果也与 SEM 和 EDS 分析结果一致。 另外, 与矿化前的牙本质相比, 实验组矿化后图谱 出现 $\mathrm{CaCO}_{3}$ 的 (104) ${ }^{[30]}$ 晶面特征衍射峰强度显著增 强, 可能是因为在人工唾液的矿化过程中, 涂覆糊 
MNBGP 剂的牙本质切片局部快速释放更高浓度 $\mathrm{Ca}^{2+}$, 形成了更多的 $\mathrm{CaCO}_{3}$ 晶体。

\section{3 结论}

以不同粒径的微纳米生物活性玻璃微球为分散 质海藻酸钠-磷酸盐缓冲溶液为分散剂, 成功制备 了便于操作的新型生物活性玻璃糊剂用于牙本质脱 敏。不同粒径微纳米生物活性玻璃微球制备的糊剂 均能与牙本质界面紧密结合, 含尺寸较小微纳米生 物活性玻璃微球的糊剂在脱矿牙本质切片表面分布 得更均匀。微纳米生物活性玻璃微球制备的糊剂在 人工唾液中能较好地诱导牙本质再矿化形成磷灰石 以堵塞封闭牙本质小管, 脱矿牙本质切片表面形成 的磷灰石层随矿化时间延长而增厚, 矿化 $28 \mathrm{~d}$ 时磷 灰石层的厚度可达到 5 10 $\mu \mathrm{m}$ 。糊剂中生物活性玻 璃微球的尺寸与暴露的牙本质小管直径相当或略小 时可以更好地填充、矿化封闭牙本质小管。本研究 证实微纳米生物活性玻璃微球材料将在牙本质脱敏 方面具有较好潜在应用前景。

\section{参考文献:}

[1] HOLLAND G R, NARHI M N, ADDY M, et al. Guidelines for the design and conduct of clinical trials on dentine hypersensitivity. Journal of Clinical Periodontology, 1997, 24(11): 808-813.

[2] ADDY M, WEST N X. The role of toothpaste in the aetiology and treatment of dentine hypersensitivity. Monogr. Oral. Sci., 2013, 23: $75-87$.

[3] ARNOLD W H, PRANGE M, NAUMOVA E A. Effectiveness of various toothpastes on dentine tubule occlusion. Journal of Dentistry, 2015, 43(4): 440-449.

[4] Berkathullah M, FAROOK M S, MAHMOUd O. The effectiveness of remineralizing agents on dentinal permeability. BioMed Research International, 2018, 2018: 4072815.

[5] BAINO F, HAMZEHLOU S, KARGOZAR S. Bioactive glasses: where are we and where are we going? Journal of Functional Biomaterials, 2018, 9(1): 25.

[6] JONES J R. Review of bioactive glass: from Hench to hybrids. Acta Biomaterialia, 2013, 9(1): 4457-4486.

[7] SKALleVOLD H E, ROKAYA D, KHURSHID Z, et al. Bioactive glass applications in dentistry. International Journal of Molecular Sciences, 2019, 20(23): 5960.

[8] LITKOWSKI L, GREENSPAN D C. A clinical study of the effect of calcium sodium phosphosilicate on dentin hypersensitivity-proof of principle. J. Clin. Dent., 2010, 21(3): 77-81.

[9] GENDREAU L, BARLOW A P, MASON S C. Overview of the clinical evidence for the use of NovaMin in providing relief from the pain of dentin hypersensitivity. J. Clin. Dent., 2011, 22(3): 90-95.

[10] HOFFMAN D A, CLARK A E, RODY W J, et al. A prospective randomized clinical trial into the capacity of a toothpaste containing NovaMin to prevent white spot lesions and gingivitis during orthodontic treatment. Progress in Orthodontics, 2015, 16(1): 25.

[11] FAROOQ I, MAJEED A, ALSHWAIMI E, et al. Efficacy of a novel fluoride containing bioactive glass based dentifrice in remineralizing artificially induced demineralization in human enamel. Fluoride, 2019, 52(3): 447-455.

[12] WANG Z, SA Y, SAURO S, et al. Effect of desensitising toothpastes on dentinal tubule occlusion: a dentine permeability measurement and SEM in vitro study. Journal of Dentistry, 2010, 38(5): $400-410$.

[13] VOLLENWEIDER M, BRUNNER T J, KNECHT S, et al. Remineralization of human dentin using ultrafine bioactive glass particles. Acta Biomater, 2007, 3(6): 936-943.

[14] CURTIS A R, WEST N X, SU B. Synthesis of nanobioglass and formation of apatite rods to occlude exposed dentine tubules and eliminate hypersensitivity. Acta Biomater., 2010, 6(9): 3740-3746.

[15] SHENG X Y, GONG W Y, HU Q, et al. Mineral formation on dentin induced by nano-bioactive glass. Chinese Chemical Letters, 2016, 27(9): 1509-1514.

[16] FERNANDES H R, GADDAM A, REBELO A, et al. Bioactive glasses and glass-ceramics for healthcare applications in bone regeneration and tissue engineering. Materials, 2018, 11(12): 2530.

[17] ZHENG K, BOCCACCINI A R. Sol-Gel processing of bioactive glass nanoparticles: a review. Advances in Colloid and Interface Science, 2017, 249: 363-373.

[18] HU Q, CHEN X, ZHAO N, et al. Fabrication and characterization of dodecylamine derived monodispersed mesoporous bioactive glass sub-micron spheres. Journal of Sol-Gel Science and Technology, 2014, 69(1): 9-16.

[19] LIANG K, GAO Y, LI J, et al. Effective dentinal tubule occlusion induced by polyhydroxy-terminated PAMAM dendrimer in vitro. RSC Advances, 2014, 4(82): 43496-43503.

[20] GAL J Y, FOVET Y, ADIB-YADZI M. About a synthetic saliva for in vitro studies. Talanta, 2001, 53(6): 1103-1115.

[21] SCHUMACHER M, HABIBOVIC P, van RIJT S. Mesoporous bioactive glass composition effects on degradation and bioactivity. Bioactive Materials, 2021, 6(7): 1921-1931.

[22] ZHANG X, ZENG D, LI N, et al. Large-pore mesoporous $\mathrm{Ca}$-Si-based bioceramics with high in vitro bioactivity and protein adsorption capability for bone tissue regeneration. Journal of Materials Chemistry B, 2016, 4(22): 3916-3924.

[23] XU Z, NEOH K G, KISHEN A. A biomimetic strategy to form calcium phosphate crystals on type I collagen substrate. Materials Science and Engineering: C, 2010, 30(6): 822-826.

[24] ZHAO F, ZHANG W, FU X, et al. Fabrication and characterization of bioactive glass/alginate composite scaffolds by a self-crosslinking processing for bone regeneration. RSC Advances, 2016, 6(94): 91201-91208.

[25] XU C, WANG X, ZHOU J, et al. Bioactive tricalcium silicate/alginate composite bone cements with enhanced physicochemical properties. Journal of Biomedical Materials Research Part B: Applied Biomaterials, 2018, 106(1): 237-244.

[26] MARELli B, GHEZZI C E, BARRALET J E, et al. Three-dimensional mineralization of dense nanofibrillar collagenbioglass hybrid scaffolds. Biomacromolecules, 2010, 11(6): 1470-1479.

[27] FAN Y, SUN Z, MORADIAN-OLDAK J. Controlled remineralization of enamel in the presence of amelogenin and fluoride. Biomaterials, 2009, 30(4): 478-483.

[28] DAI L L, MEI M L, CHU C H, et al. Remineralizing effect of a new strontium-doped bioactive glass and fluoride on demineralized enamel and dentine. Journal of Dentistry, 2021, 108: 103633.

[29] WU Y, TANG L, ZHANG Q, et al. A novel synthesis of monodispersed bioactive glass nanoparticles via ultrasonic-assisted surfactant-free microemulsion approach. Materials Letters, 2021, 285: 129053 .

[30] NIRMALA DEVI M, SANJIV RAJ K, SUBRAMANIAN V K. Synergistic effects of magnesium and EDTA on polymorphism and morphology of $\mathrm{CaCO}_{3}$ and its influence on scale. Journal of Crystal Growth, 2021, 564: 126108. 